Historic, Archive Document

Do not assume content reflects current scientific knowledge, policies, or practices. 

Tree, Shrub, Perennial 爱 and Fruit Seeds 采

For Ornamental and Foresty Purposes

\section{Price List 1921-1922}

\section{CONYERS B. FLEU, Jr. Tree Seedsman}

GERMANTOWN - PHILADELPHIA, U. S. A. 


\section{Tree, Shrub and Perennial Seeds}

PRACTICAL PLANTING INSTRUCTIONS. Carefully select a location for your seed beds, if possible, from heavy east and northeast winds, and provide for windbreaks in the form of hedges, plantings, fence, etc. The soil should be of a well drained and sandy loam. If the soil is heavy, add sand; if sandy, it can be improved by adding loam to it. Well-rotted manure or compost can be used to enrich the soil, but avoid the use of fresh manure. The soil should be thoroughly spaded or ploughed to a depth of at least one foot and harrowed or raked until the soil is in fine condition.

The seed beds should be three feet wide and sow the seed broadcast; after sowing, cover to the depth of about the thickness of the seed with light sandy soil and gently press the beds with the back of a spade to bring the earth in contact with the seeds. Shade is an essential, as seeds germinate best in darkness; this can be accomplished by either making a lath sash or a light covering with forest leaves or very well-rotted manure. The lath sash is, however, the best. This can be made with building laths made into handy frames and fastened on post about I8 inches above the ground. The leaves, manure or sash should be removed as soon as the seedling appears, as light is needed.

The seeds of Conifers and Evergreen Trees should be sown in the spring and those of a Deciduous nature in the fall. To preserve seeds until the proper planting season: Mix the Conifer and Evergreen seeds in dry sand and the Deciduous seeds, that cannot be planted in the fall, in slightly moist soil; keep the soil in a damp condition until ready to sow in the spring.

Seeds which have hard shells such as Gymnocladus, honey and black locust. if not sown in the fall, are best soaked for a day or so and then at once sown.

NOTICE: Every effort is extended to secure and collect only the very best seeds, but it is known that some seeds are occasionally wormy, such as Quercus and Castanea. This defect, however, cannot be detected until after the worm has eaten its way out, which often is after the seeds have been shipped. While on the other hand, there are trees such as Liriodendron, Sugar Maple, etc., that never perfect all their seeds. The imperfect seeds cannot be separated. With this in view and the many known and unknown causes which prevent seeds from germinating, all seeds are sold without any warranty, expressed or implied, as to quality, productiveness or any other matter of any seeds, and in no way will I be responsible for crop conditions. All orders are accepted on these conditions. Should any cause for complaint arise on receipt of shipment notice must be sent within one week of same, otherwise it is understood that the goods are accepted.

CONDITIONS. As I collect but few more seeds than are ordered, all offers and acceptances of orders are subiect to the condition of the season's crop, and failing to get the seeds expected or the quantities ordered from my growers and collectors, I reserve the right to reduce proportionately all orders that have been booked.

SHIPPING FACILITIES. Small orders can be sent to all parts of the world by sample post and to nearly every country by parcel post. Shipments are dispatched very quickly owing to my warehouse being closely connected with the forwarders of New York and Philadelphia ports. The cost of postage, bags and boxes is charged additionally.

TERMS. My terms are cash with order or satisfactory reference must be supplied.

RATES. One-quarter pound sold at the pound rate, ten pounds or over at the twenty-five pound rate and twenty-five pounds or over at the one hundred pound rate.

\section{Conyers B. Fleu, Jr. TREE SEEDSMAN Germantown, Philadelphia, U. S. A.}




\begin{tabular}{|c|c|c|c|c|c|c|c|}
\hline Abies & (Picea) & Amabilis, California Fir . . & $\$ .50$ & $\$ 5.00$ & $\$ 4.75$ & $\$ 4.50$ & \\
\hline ، & & Balsamea, Balsam Fir .. & .35 & 3.00 & 2.75 & 2.65 & 2.50 \\
\hline “6 & “" & Brachyphylla $\ldots \ldots \ldots \ldots \ldots \ldots \ldots$ & .35 & 3.50 & 3.35 & 3.25 & 5 \\
\hline “6 & 66 & Cephalonica, Cephaloniana Fir ..... & .25 & 2.50 & 2.25 & 2.00 & \\
\hline "6 & ، & Cilicica, Cicilian Fir & .30 & 3.00 & 2.75 & 2.50 & 2.25 \\
\hline 66 & 66 & Concolor, California Silver Fir ..... & .25 & 3.00 & 2.75 & 2.65 & 00 \\
\hline "6 & "، & Concolor, Colorado Silver Fir ....... & .25 & 3.00 & 2.90 & 30 & \\
\hline 66 & 66 & Firma, Japanese ............... & .35 & 2.00 & 1.75 & 1.65 & 1.50 \\
\hline 66 & 66 & Frasteri $\ldots \ldots \ldots \ldots \ldots$ & .50 & 4.50 & 4.25 & & \\
\hline 66 & 66 & Grandis, Pacific Coast Fir ........ & .40 & 4.00 & 3. & 3.50 & \\
\hline “6 & 66 & Homolepis, Japan ................ & .40 & 4.00 & 3.75 & 3.50 & \\
\hline “" & 66 & Magnifica, Pacific Coast Fir ...... &. \pm 0 & 4.00 & 3.75 & 3.50 & \\
\hline "، & 66 & Mariesii, Japan Silver Fir ......... & .50 & 0 & 4.25 & 4.00 & \\
\hline "“ & “" & Nobilis, Pacific Coast Fir ........ & .50 & 4. & 4. & 4. & \\
\hline 6" & "6 & Nordmanniana, Nordman Fir....... & 0 & 3.00 & 2. & 2.75 & 2 \\
\hline "“ & "6 & Pectinata, European Silver Fir .... & .25 & 1.50 & 1.35 & 1.25 & 1. \\
\hline 66 & 66 & Sachalinensis, Saghalien Fir ...... & .75 & 6.50 & 5.85 & 5.75 & 5.6 \\
\hline "6 & 6 & $\ldots \ldots \ldots \ldots$ & & 5.00 & 4.75 & 4.65 & 4.50 \\
\hline 66 & 66 & ..... & & & 4.75 & 4.65 & 4.5 \\
\hline 6 & & & & & 4.75 & & \\
\hline
\end{tabular}

Araucaria Excelsa, Norfolk Island Pine ........100 Seeds $\$ 2.00 ; 1000$ Seeds $\$ 15.00$ Imbricata, Chilian Pine Tree .......100 Seeds $\$ 2.00 ; 1000$ Seeds $\$ 15.00$

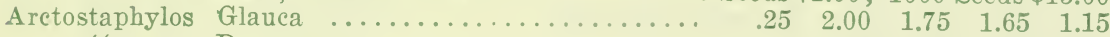

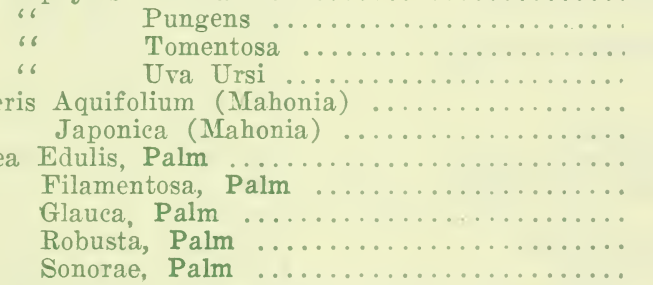

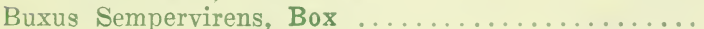

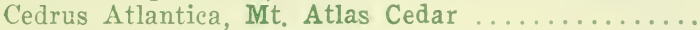

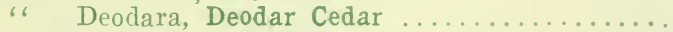

" Libani, Cedar of Lebanon

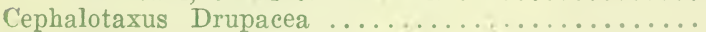

" Fortunei .............................

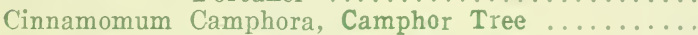

Crataegus Pyracantha, Evergreen Thorn ...........

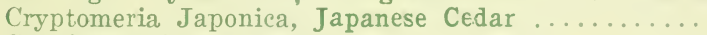

Cunninghamia Lancellata

$$
\text { Sinensis }
$$

$\begin{array}{rrrrr}.25 & 2.00 & 1.75 & 1.65 & 1.50 \\ .25 & 2.00 & 1.75 & 1.65 & 1.50 \\ .50 & 4.00 & 3.75 & & \\ .25 & 2.00 & 1.75 & 1.50 & 1.35 \\ .50 & 4.50 & 4.35 & 4.25 & \\ .15 & 1.25 & 1.15 & 1.00 & \\ .15 & 1.25 & 1.15 & 1.00 & \\ .25 & 2.00 & 1.85 & 1.75 & \\ .25 & 2.00 & 1.85 & 1.75 & \\ .25 & 2.00 & 1.85 & 1.75 & \\ .25 & 2.00 & 1.85 & 1.75 & \\ .25 & 2.50 & 2.25 & 2.15 & 2.00 \\ .25 & 2.50 & 2.25 & 2.15 & 2.00 \\ .25 & 2.50 & 2.25 & 2.15 & 2.00 \\ .25 & 2.00 & 1.85 & 1.75 & \\ .25 & 2.00 & 1.85 & 1.75 & \\ .25 & 1.25 & 1.00 & .75 & \\ .15 & 1.50 & 1.25 & 1.15 & 1.10 \\ .25 & 2.00 & 1.85 & 1.75 & \\ .25 & 2.50 & 2.35 & 2.25 & \\ .00 & 10.00 & & & \\ .35 & 3.00 & 2.90 & 2.75 & 2.50 \\ .25 & 2.50 & 2.35 & 2.25 & \\ .35 & 3.50 & 3.35 & 3.25 & \\ .50 & 5.00 & 4.75 & 4.50 & 4.35 \\ .35 & 3.50 & 3.35 & 3.25 & \\ .35 & 3.00 & 2.75 & 2.50 & 2.25 \\ .50 & 15.00 & & & \\ .25 & 2.00 & 1.85 & 1.75 & \\ .00 & & & & \\ .25 & 2.00 & 1.90 & 1.85 & 1.75 \\ .50 & 4.00 & 3.90 & 3.75 & 3.65 \\ .25 & 2.00 & 1.85 & 1.75 & \\ .25 & 2.50 & 2.25 & 2.00 & 1.75 \\ .15 & .75 & .65 & .55 & .50 \\ . .5 & 4.00 & 3.75 & 3.50 & \\ & & & & \end{array}$

Garrya Elliptica............................ 1.0

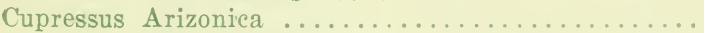

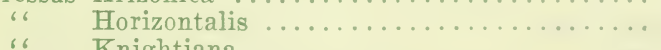

“ Knightiana

" Lawsoniana, Lawson's Cypress .........

، Lindleyi

" Macrocarpa, Monterey Cypress ...........

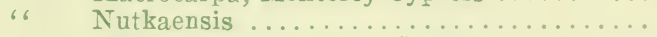

“ Sempervirens, Italian Cypress

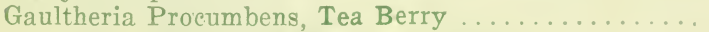

" Shallon, Western Tea Berry

Ilex Aquifolium, English Holly

"Crenata, Japan Holly

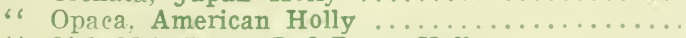

* Sicboldii, Japan Rcd-Berry Holly

5 lbs. 25 lbs. 100 lbs,

per oz. perlb. price price price,
per $1 \mathrm{~b}$. per lb. per lb.

per oz. perlb. price price price
per $l b$. per $1 \mathrm{~b}$. per $\mathrm{lb}$. $.50 \$ 5.00 \quad \$ 4.75 \$ 4.50$

$\begin{array}{llll}.40 & 4.00 & 3.75 & 3.50\end{array}$

$\begin{array}{llll}.50 & 4.50 & 4.25 & 4.00\end{array}$

$4.35 \quad 4.25$

$.25 \quad 1.50$

.00

5.65

.50 , 


\section{Juniperus}

66
66
66
66
66
66
66
66
66
66
66
66
66

Kalmia

Californica

Chinensis, Chin

Communis Nan

Littoralis, from

Monosperma

Pinchoti

Prostrata

Rigidia, Japanese Cedar .............

Sabina, Savin Juniper...

Sabinoides

Scopulorum

Suecica, Swedish Juniper

Virginiana, Red Cedar

"Latifolia, Sheep Laurel

Larix Americana, American Larch

" Europaea, European Larch

“ Leptolepsis, Japanese Larch . .

" Occidentalis, Western Larch ."

“ Sibirica, Siberian Larch

Laurus Nobilis, Sweet Bay

Libocedrus Decurrens

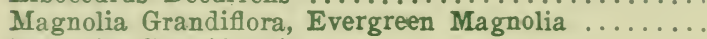

Nuttalia Cerasiformis

Oreodaphne Californica, California Nutmeg .......

Photinia Arbutifolia

Picea (Abies) Ajanensis

$\begin{array}{ll}66 & 66 \\ 66 & 66 \\ 66 & 66 \\ 66 & 66 \\ 66 & 66 \\ 66 & 66 \\ 66 & 66 \\ 66 & 66 \\ 66 & 66 \\ 66 & 66 \\ 66 & 66 \\ 66 & 66 \\ 66 & 66 \\ 66 & 66\end{array}$

Alba, White Spruce ..............

Alcoquiana, Japanese Spruce

Canadensis, Black Hill Spruce ......

Engelmanni

Excelsa, Norway Spruce

Glehnii, from Japan

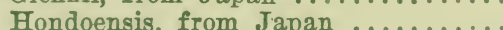

Koyami, from Japan .............

Nigra, Black Spruce ..............

Orientalis, Oriental Spruce

Polita ......................

Pungens, Colorado Blue Spruce .....

Rubra, Red Spruce ..............

Sitchensis (Menziesii)

Pinus Armandi, from China $\ldots . . . . . . . . . . . .$.

Austriaca, Austrian Pine ................

Banksiana, Jack Pine

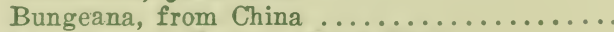

Canariensis, Canary Island Pine ............

Carribaea, Calabrian Pine ...............

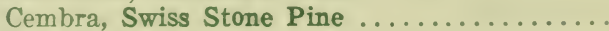

Contorta

Coulteri, Big Cone Pine

Cubensis, Cuban Pine

Densiflora, Japanese Pine

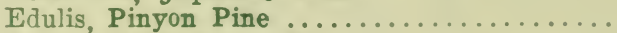

Excelsa, Himalayan Pine

Flexilis, Colorado Pine

Halepensis, Aleppo Pine

Insignis, Monterey Pine

Jeffrey

Koraiensis
5 lbs. 25 lbs. 25 lbs.

price price price

per $\mathrm{lb}$. per lb. per lb.

\section{$.35 \quad 3.00$}

2.85

2.75

$\begin{array}{ll}.35 & 3.00\end{array}$

$.25 \quad 1.50$

$.25 \quad 2.00$

$\begin{array}{ll}.50 & 5.00\end{array}$

$.15 \quad 1.50$

$.35 \quad 3.50$

$.25 \quad 1.50$

.50

.25

.35

.25

.25

.15

.75

.75

2.00

.40

.50

.75

.25

.35

.25

.35

.20

.15

1.00

.50

.50

.50

.40

.35

5.00

2.50

3.00

2.00

2.00

1.00

2.85

1.35

1.85

4.75

1.35

3.35

1.35

4.75

2.35

2.75

1.75

1.85

5.00

5.00

20.00

3.00

5.00

7.50

4.00

\subsection{0}

2.75

$4.75 \quad 4.50$

2.50

7.35

7.25

4.25

2.00

3.85

3.75

3.50

1.85

1.75

2.00

3.35

3.25

3.00

1.85

1.75

2.00

$\begin{array}{ll}2.75 & 2.75\end{array}$

1.25

1.75

1.50

$1.15 \quad 1.10$

1.00

10.00

9.50

9.00

5.50

5.35

5.25

5.00

6.00

5.85

5.75

4.00

3.75

5.75

5.50

$.35 \quad 2.50$

2.40

3.50

3.25

$\begin{array}{lll}1.00 & 10.00\end{array}$

9.50

2.35

2.25

$50 \quad 5.00$
1.50

$4.75 \quad 4.50$

$1.50 \quad 15.00$

$\begin{array}{ll}.50 & 5.00\end{array}$

$4.75 \quad 4.50$

$.50 \quad 4.50$

4.25

4.00

.50

.50

.50

.75

.35

.30

.60

.35

.35

.35

$$
.25
$$

5.00

4.75

4.50

4.00

3.90

3.85

5.00

4.75

4.50

6.50

6.35

6.25

3.00

2.75

2.50

3.00

2.90

2.85

5.65

6.00

5.75

2.50

3.00

2.75

2.50

3.00

2.75

2.50

2.00

1.75

1.50

5.50

5.35

5.25

4.25

4.00

3.75

3.50

2.50

2.25

2.00

2.50

2.35

2.25

3.00

2.85

2.75

3.00

2.75

2.50

3.00

2.75

2.50

4.00

3.75

3.50

$\begin{array}{llll}.30 & 3.00 & 2.75 & 2.50\end{array}$
3.25

\subsection{5}

6.00

2.75

5.50

1.25

5.00

4.00

1.75

2.50 
Pinus Lambertiana, Sugar Pine

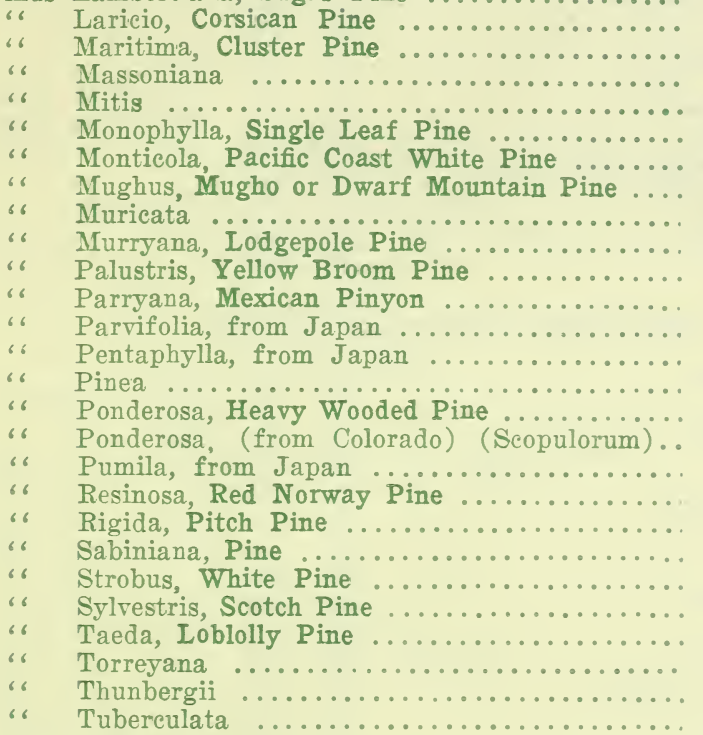

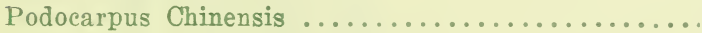

Prunus (Cerasus) Caroliniana

$$
\text { “ } 6 \text { " I } \quad \text { Integrifolia, Entire Leaf Cherry... }
$$

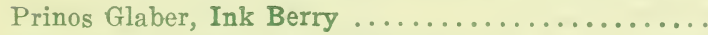

Pseudotsuga Douglasii, Douglas Spruce (Ore. variety)

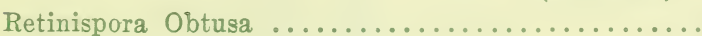

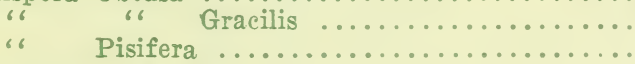

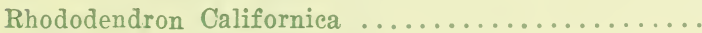

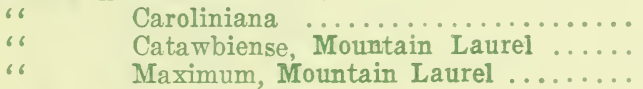

Sciadopitys Verticillata, Umbrella Pine ...........

Sequoia Gigantea, California Big Tree

"Sempervirens, California Redwood

Taxus Baceata, English Yew

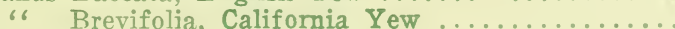

“Cuspidata, Japanese Yew

Thuya Gigantea, Western Arbor Vitae ............

“ Occidentalis, American Arbor Vitae .........

“Orientalis, Chinese Arbor Vitae

" Orientalis Aurea, Chinese Golden Arbor Vitaf

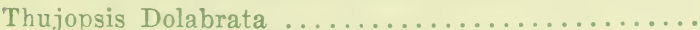

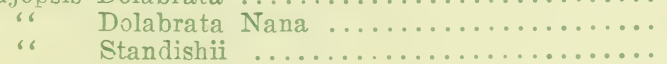

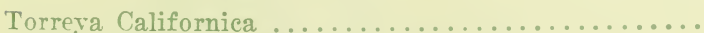

\begin{tabular}{|c|c|c|c|c|}
\hline & & & & \\
\hline 5 & 2.50 & 2.35 & 2.25 & 2 \\
\hline & 3.50 & 3.35 & 3.25 & 3.00 \\
\hline 15 & 1.50 & 1.35 & 1.25 & \\
\hline .25 & 2.00 & 1.85 & 1.75 & \\
\hline & 4.50 & 4.35 & 4.25 & 4.1 \\
\hline & 3.5 & 3.35 & 3.25 & 3. \\
\hline & 5.5 & 5.35 & 5.25 & 5.1 \\
\hline & 3.5 & 3.35 & 3.25 & 3. \\
\hline & 6.00 & 5.85 & 5.75 & \\
\hline & 6.0 & 5.75 & 5.65 & \\
\hline & 2.0 & 1. & 1.75 & 1 \\
\hline & 4.5 & 4.35 & 4.25 & 4.15 \\
\hline & 4.8 & 4.25 & 4.00 & \\
\hline & 4. & 3.75 & 3.75 & 3.5 \\
\hline & 1. & 1.00 & .85 & \\
\hline & 3 & 2.85 & 2.75 & \\
\hline & 2.5 & 2.35 & 2.25 & 2.15 \\
\hline 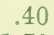 & 4.00 & 3.85 & 3.75 & \\
\hline 50 & 15.00 & 14.50 & 14.00 & 13.50 \\
\hline & 5.0 & 4.75 & 4.50 & \\
\hline & 1.75 & 0 & 1.35 & 1.2 \\
\hline & 3.5 & 3. & 3.00 & 2.7 \\
\hline & 3.00 & 2. & 2.75 & 2. \\
\hline & 5.0 & 4.85 & 4.75 & 4. \\
\hline & $4 .($ & 3.85 & 3.75 & 3.5 \\
\hline .2 & 2 & 5 & 2.00 & 1.7 \\
\hline .50 & 5.00 & 4.85 & 4.75 & 4.50 \\
\hline .25 & 2.50 & & & \\
\hline .1 & 1.00 & .85 & .75 & \\
\hline & 1.75 & 1.65 & 1.50 & \\
\hline .15 & 1.75 & 1.65 & 1.50 & \\
\hline .15 & 1.25 & 1.00 & .90 & .8 \\
\hline .5 & 4.00 & 3.90 & 3.75 & 3. \\
\hline 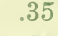 & 3 & 3.35 & 3.25 & \\
\hline .25 & 3.00 & 2.75 & 2.50 & \\
\hline 1. & 10. & & & \\
\hline & 3.00 & 2.75 & 2.50 & \\
\hline & & & & \\
\hline .70 & $\begin{array}{l}7.00 \\
5.00\end{array}$ & & & \\
\hline .75 & 5.00 & & & \\
\hline .35 & 3.50 & 3.25 & 3.00 & \\
\hline & 7.50 & 7.35 & 7.25 & 7.0 \\
\hline 40 & 4.00 & 3.75 & 3.50 & 3.2 \\
\hline & 2.00 & 1.85 & 1.75 & \\
\hline 0 & 12.00 & & & \\
\hline .35 & 3.00 & 2.75 & 2.50 & \\
\hline & 5.00 & 4.75 & 4.50 & $4 .{ }^{\circ}$ \\
\hline & 4.00 & 3.75 & 3.50 & 3.2 \\
\hline & 1.00 & & 05 & .75 \\
\hline 30 & 3.00 & 2.85 & 2.75 & \\
\hline & 8.50 & & & \\
\hline & & & & \\
\hline & 7 & & & \\
\hline & 2.8 & & & \\
\hline & 2 & & 1. & \\
\hline & 0.00 & & & \\
\hline
\end{tabular}

Nucifera, from Japan

Tsuga Canadensis, Hemlock Spruce

5 lbs. 25 lbs. 100 lbs.

per oz. per lb. price price price per lb. per lb. per lb. 

5 lb. 25 lbs, $100 \mathrm{lbs}$ price price price per lb. per lb. per lb.

Tsuga Diversifolia, Japanese Hemlock

“ Mertensiana, Western Hemlock

$\begin{array}{ll}.75 & 7.50\end{array}$

1.0010 .00

1.0010 .00

“Sieboldi, Japanese Hemlock

Ulex Europaea, Furze

Yucca Angustifolia, Adam's Needle

“ Baccata, Adam's Needle

" Brevifolia, Adam's Needle

“Filamentosa, Adam's Needle

“ Radiosa, Adam's Needle

"Whipplei, Adam's Needle $\begin{array}{ll}.75 & 7.50\end{array}$

$.25 \quad 2.25$

$.50 \quad 4.50$

1.0010 .00

$.25 \quad 2.50$

1.0010 .00

$.50 \quad 4.50$
$.25 \quad 2.00$

$\begin{array}{lll}1.85 & 1.75 & 1.50\end{array}$

$\begin{array}{lll}2.15 & 2.10 & 2.00\end{array}$

$2.25 \quad 2.00$

DECIDUOUS TREES AND SHRUBS

Acer Campestre, English Maple $\ldots \ldots \ldots \ldots \ldots . . . \ldots$

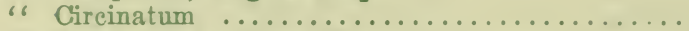

$\begin{array}{lll}.15 & 1.00 \quad .85\end{array}$

.75

$\begin{array}{lll}.35 & 3.00 & 2.75\end{array}$

" Dasycarpum, Silver Maple ..............

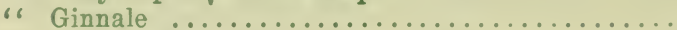

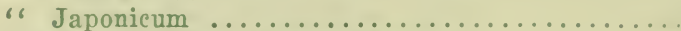

“ Macrophyllum, Oregon Maple ............

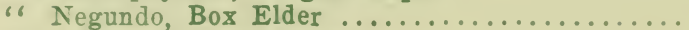

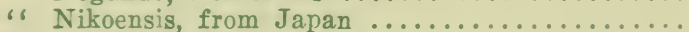

“ Palmatum, Japanese Maple ...............

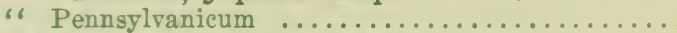

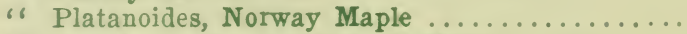

“Pseudo-Platanus, Sycamore Maple ...........

“ Rubrum, Red Maple ..................

“ Saccharinum, Sugar Maple ..............

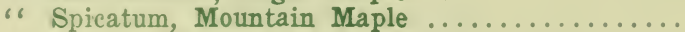

“ Striatum, Striped Maple ................

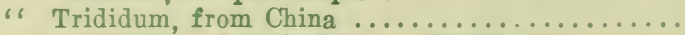

Aesculus Glabra, American Horse-Chestnut

$\begin{array}{llll}.25 & 1.00 & .85\end{array}$

$\begin{array}{lll}.25 & 2.00 & 1.90\end{array}$

.252 .00

$.25 \quad 1.50$

$.15 \quad .75$

1.35

2.50

$.40 \quad 4.00$

.65

.75

1.80

1.75

1.251 .15

$.60 \quad .50$

.25

.25

.25

.25

.25

.35

.35

.25

.15

.15

.25

.25

.25

.50

.15

.40

.35

.35

.15

.15

.50

.50

.50

.50

.50

.50

.50

.35

2.50

.85

3.75

3.50

$2.35 \quad 2.25$

.75

.85

2.50

.75

2.35

$1.50 \quad 1.35$

$2.50 \quad 2.35$

$2.50 \quad 2.35$

2.00

.75

.75

.75

3.00

1.50

6.00

1.00

4.00

3.00

3.00

1.00

.75

1.75

.65

.65

.65

.65

.65

2.25

1.25

2.25

2.25

1.50

.50

.50

.60

2.15

.50

.50

2.15

1.15

2.15

2.15

$1.25 \quad 1.15$

1.10

$\begin{array}{ll}.75 & .50\end{array}$

.40

$\begin{array}{ll}3.75 & 3.50\end{array}$

$2.75 \quad 2.50$

2.25

$2.75 \quad 2.50$

$.90 \quad .85$

$.65 \quad .60$

.50

7.00

5.00

7.00

7.00

7.00

5.00

5.00

3.50

7.50

2.00

$3.25 \quad 3.00$

3.00

$\begin{array}{lll}1.75 & 1.50 & 1.25\end{array}$

$\begin{array}{lll}2.75 & 2.50 & 2.25\end{array}$

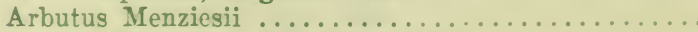

Ardisia Crenulata, from Japan

7.50

7.50

$.75 \quad 7.50$

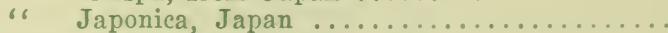

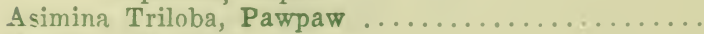

Aucuba Japonica 


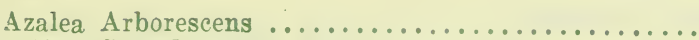

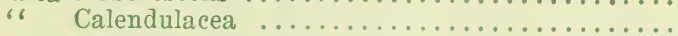

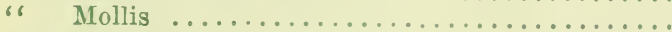

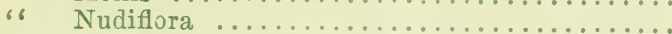

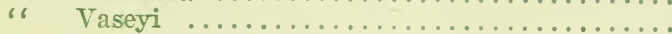

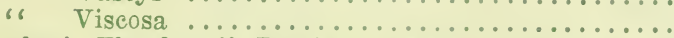

Berberis Thunbergii, Berries, Japanese Barberry .... " " Clean Seed, Japanese Barberry.

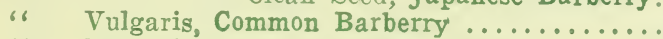

“Vulgaris Purpurea, Purple Barberry .......

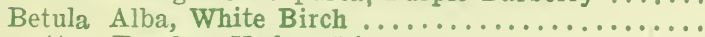

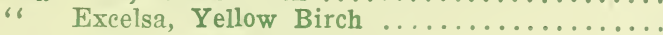

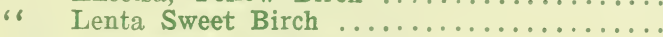

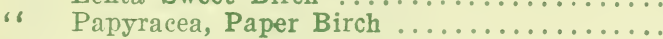

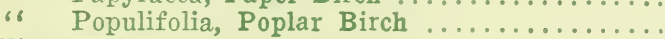

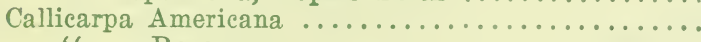

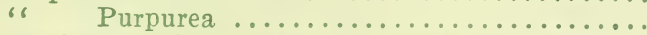

Calycanthus Floridus Laevigatus, Sweet Shrub ..... " Occidentalis, Western Shrub .........

Camellia Japonica .......................

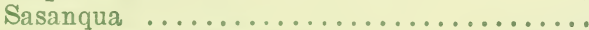

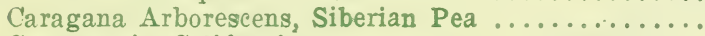

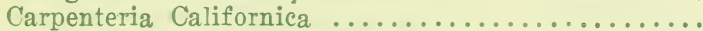

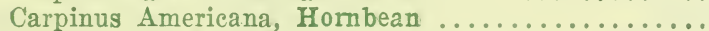

" Betulus, European Hornbean ...........

“Yedoensis, Japanese Hornbean ............

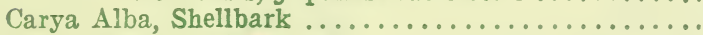

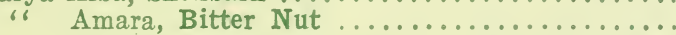

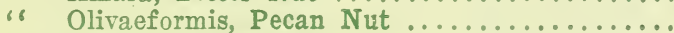

“ Porcina, Pig Nut ....................

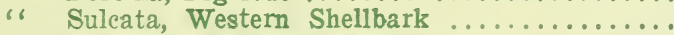

" Tomentosa, Hickory Nut ..............

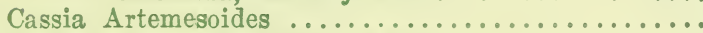

" Marilandica, Wild Senna ..............

Castanea Japonica, Japanese Chestnut ...........

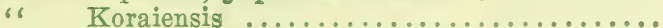

Catalpa Bignonioides, Eastern Catalpa ...........

"Speciosa, Western Catalpa .............

This is the true forest tree speciosa, guara

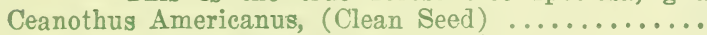

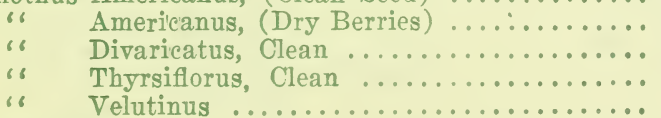

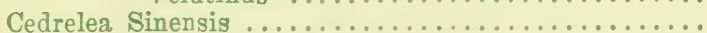

Celtis Chinensis, Chinese Nettle Tree ............

" Occidentalis, Nettle Tree ................

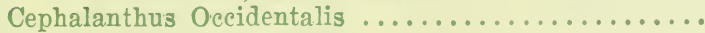

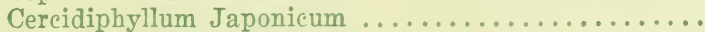

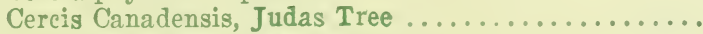

" Chinensis, Japonicum ................

"Occidentalis, Western Judas ............

Chionanthus Virginica, White Fringe (Dry Berries).

Citrus Trifoliata, Hardy Orange (Clean Seed).

Citrus Trifoliata, Hardy Orange ..............

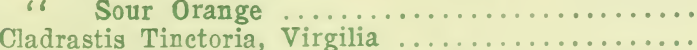

Clethra Alnifolia, Sweet Pepper Bush ..........

Colutea Arborescens

5 lbs. 25 lbs, 100 lbs. price price price per lb. per lb. per lb.

$\begin{array}{ll}.75 & 7.50\end{array}$

$\begin{array}{ll}.75 & 7.50\end{array}$

1.0010 .00

$\begin{array}{ll}.75 & 7.50\end{array}$

$.75 \quad 7.50$

$.75 \quad 7.50$

$.25 \quad 1.50$

$\begin{array}{ll}.75 & 5.00\end{array}$

$.15 \quad 1.25$

$.25 \quad 2.00$

$.10 \quad 1.00$

$.25 \quad 2.00$

$.25 \quad 2.00$

.252 .00

.25

.15

.15

.15

.35

.30

.30

.25

2.00

.15

.15

.40

$15 \quad .25$

.25

.75

.25

.25

.25

1.0010 .00

$.15 \quad 1.25$

.75

.75

.15

.25

1.50

1.50

teed

.353 .50

.25

1.00

2.00

1.0010 .00

$50 \quad 5.00$

1.00

1.0010 .00

$.15 \quad 1.50$

$.15 \quad 1.00$

$.15 \quad .75$

1.0010 .00

$.25 \quad 1.50$

$.75 \quad 7.50$

$.35 \quad 3.50$

.251 .50

.35

.15

.15

.35

.25

.15
1.451 .35

1.25

1.151 .00

1.751 .50

$.90 \quad .85$

$1.90 \quad 1.85$

$1.90 \quad 1.85$

$1.90 \quad 1.85$

$1.35 \quad 1.25$

.85

.85

.65

3.25

2.75

2.75

1.90

.75

.75

.55

3.15

2.50

2.50

1.75

1.50

\section{.90}

.80

3.75

.20

.20

.65

.20

.20

.20

.75

3.50

.18

.18

.50

.18

.18

.18

.75

1.75

1.75

1.75

1.15

.50

3.00

1.151 .00

$\begin{array}{ll}.60 & .55\end{array}$

$.60 \quad .55$

$1.35 \quad 1.25$

.75

.15

.15

.45

.15

.15

.15

$1.40 \quad 1.35$

name.

$\begin{array}{lll}3.35 & 3.25 & 3.15\end{array}$

$\begin{array}{lll}1.85 & 1.75 & 1.65\end{array}$

.50

.50

1.25

3.15
.65

$1.35 \quad 1.25$

$.90 \quad .85$

$.70 \quad .65$

.7

$\begin{array}{lll}1.40 & 1.35 & 1.25\end{array}$

$3.35 \quad 3.25$

$1.25 \quad 1.15$

$2.25 \quad 2.15$

$\begin{array}{ll}1.35 & 1.25\end{array}$

$\begin{array}{lll}1.50 & 1.35 & 1.25\end{array}$

$\begin{array}{lll}3.50 & 3.35 & 3.25\end{array}$

$\begin{array}{lll}2.00 & 1.75 & 1.65\end{array}$

1.50 


\section{DECIDUOUS TREES AND SHRUBS (Continued)}

per oz. per lb. price price price per lb. per lb. per lb.

Cornus Alternifolia, Blue-Berried Dogwood Canadensis “ Canadensis..$\ldots \ldots \ldots \ldots \ldots \ldots \ldots \ldots \ldots \ldots$

Large White

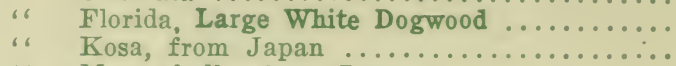

“ Macrophylla, from Japan .............

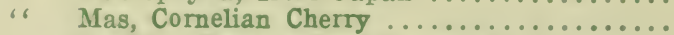

“ Nuttalli, Pacific Coast Dogwood ...........

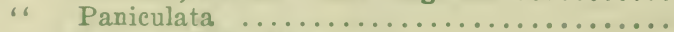

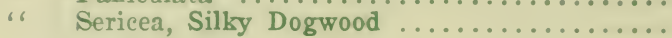

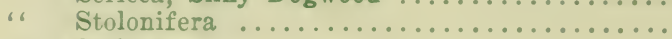

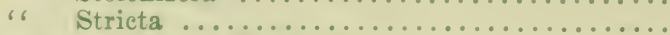

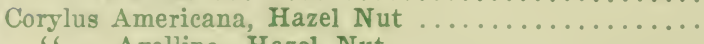

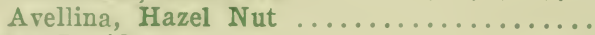

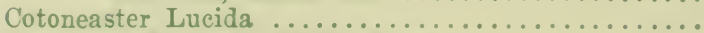

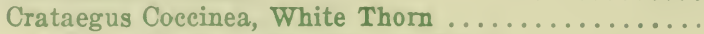

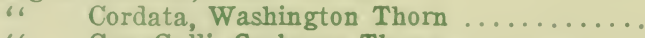

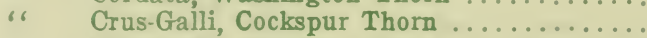

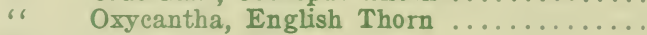

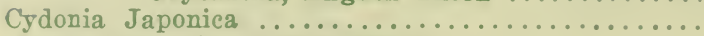

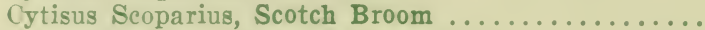

Diosprros Kaki, Japanese Persimmon ............

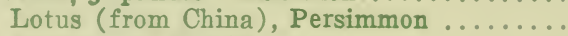

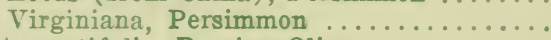

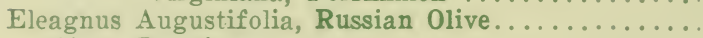

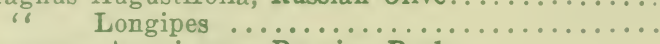

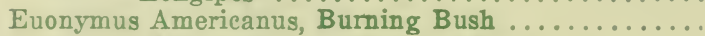

Atropurpureus, Burning Bush

" Europaeus, European Burning Bush

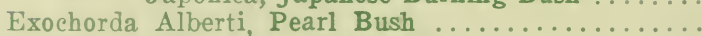

" Grandiflora, Pearl Bush .............

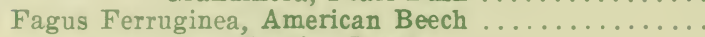

Sylvatica, English Beech

Sylvatica Purpurea, Copper Beech

Fraxinu Americana A

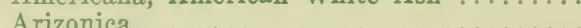

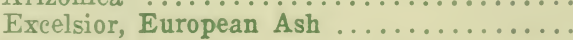

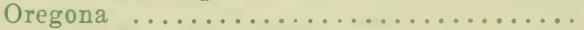

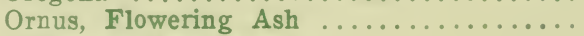

Pubescens, Red Ash .

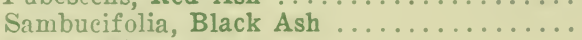

Viridis, Green Ash .....

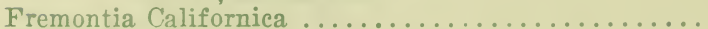

Garrya Elliptica ........................

Triacanthos, Honey Locust

Gymnocladus Canadensis, Kentucky Coffee ...............

Halesia Diptera, Snowdrop Tree

Tetraptera, Snowdrop Tree

Hamamelis Virginica, Witch Hazel

Hibiseus Syriacus, Althea

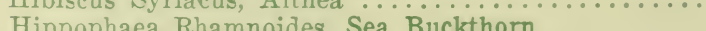

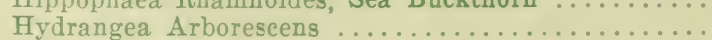

Itea Virginica

Juglans Californica, California Walnut

Cinera, Butternut

Cordiformus, Japanese Walnut

Nigra, Black Walnut
.15

.35

.15

.15

.25

.50

.15

.25

.15

.15

.15

.15

.15

.15

.15

.15

.15

.15

.15

.35

.15

.25

.25

.15

.15

.15

\section{.25}

.25

.25

.40

.35

.25

.15

.15

.35

.15

.25

.15

$$
.35
$$

.15

1.00
1.00

1.00

3.50

1.50

.75

5.00

1.00

2.00

1.00

1.00

1.00

1.50

.75

.75

1.50

.75

1.00

.75

.75

3.50

1.25

2.00

2.00

1.00

1.25

1.00

2.00

2.00

2.00

1.35

1.151 .00

$1.85 \quad 1.75$

$\begin{array}{ll}1.75 & 1.50\end{array}$

1.15

$\begin{array}{ll}1.85 & 1.75\end{array}$

1.85

3.00

3.00

2.50

1.25

1.00

3.00

1.00

1.50

1.00

3.00

1.00

3.00

2.00

.15

.15

.15

.15

.25

.15

.15

.20

1.50

.75

1.50

1.00

2.50

1.00

1.50

2.00

3.00

.50

.25

.75

.15
.90

3.35

.80

3.25

$\begin{array}{ll}1.35 & 1.25\end{array}$

$\begin{array}{ll}.65 & .50\end{array}$

.40

.85

1.75

.85

.85

.85

.75

1.65

.75

.75

.75

1.50

.50

.50

.65

.65

.65

.55

.50

.50

1.25

$\begin{array}{ll}.65 & .55\end{array}$

.75

.65

.55

.65

.55

$\begin{array}{ll}3.35 & 3.25\end{array}$

$\begin{array}{ll}.90 & .85\end{array}$

1.00

$.95 \quad .90$

1.50

1.75

1.85

1.75

2.75

2.25

2.75

2.25

1.15

2.50

1.00

.95

.90

2.85

2.75

.90

.75

. .95

1.25

2.75

.95

2.65

2.75

.90

1.90

2.65

1.85

.55

2.5

.50

1.25

1.00

.65

.60

.55

1.25

75

.45

.50

.50

.50

.75

.75

.85

2.50

.85

2.50

.85

2.35

1.75

2.25

.75

$\begin{array}{ll}1.35 & 1.25\end{array}$

$1.75 \quad 1.50$

2.852 .75

$.45 \quad .40$

2.65

$\begin{array}{lll}.20 & .18 & .15\end{array}$

$\begin{array}{lll}.65 & .55 & .50\end{array}$

$\begin{array}{lll}.12 & .10 & .08\end{array}$ 
5 lbs. 25 lbs. 100 lbs. per oz. per lb, price price price

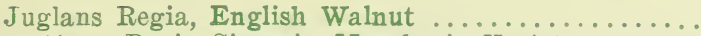
“ Regia Sinensis, Manchuria Variety ....... " Sieboldii, Japan Walnut ..............

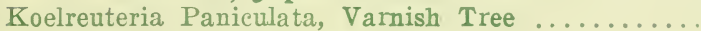

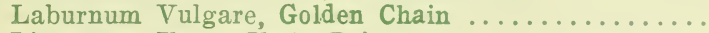

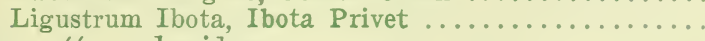

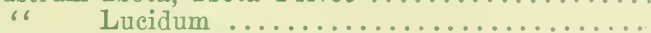

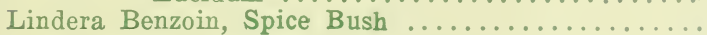

Liquidambar Formosana, from China ............ “ Styraciflua, Sweet Gum ............

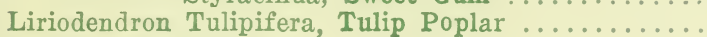

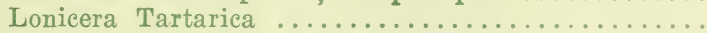

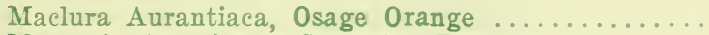

Magnolia Acuminata, Cucumber Tree ............

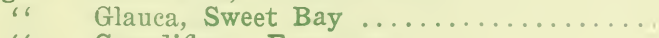
"6 Grandiflora, Evergreen

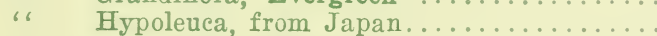

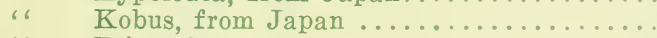
66

Tripetala

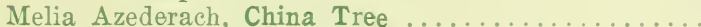

Morus Alba, White Mulberry

"6 Rubra, Red Mulberry

“ Tartarica, Russian Nulberry

Myrica Cerifera, Wax Myrtle .....

Nandina Domestica, from Japan ...............

Negundo Aceroides, Box Elder

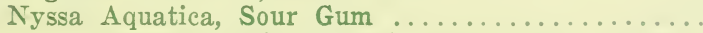

" Multiflora, (Sylvatica), Sour Gum .........

“ Uniflora, Tupelo, Sour Gum

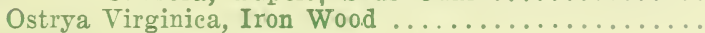

Oxydendrum Arboreum, Sour Wood per lb. per lb. per Ib.

Paulownia Imperialis, Empress Tree

.65

.50

.45

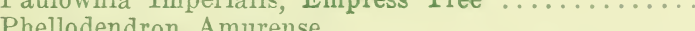

Pittosporum Tobira, from Japan $\ldots \ldots \ldots \ldots \ldots$

Platanus Occidentalis, American Plane ...........

1.00

2.50

8

2.50

2.00

2.00

3.00

3.00

2.00

1.00

.85

.75

8.85

2.75

2.35

.75

2.25

$.85 \quad .80$

$\begin{array}{ll}2.35 & 2.25\end{array}$

$\begin{array}{ll}1.75 & 1.65\end{array}$

$\begin{array}{ll}1.85 & 1.75\end{array}$

$2.85 \quad 2.75$

$2.85 \quad 2.75$

$\begin{array}{ll}1.85 & 1.75\end{array}$

4.00

.85

3.75

.75

4.00

3.75

3.50

3.00

2.85

3.50

2.75

$\begin{array}{ll}1.35 & 1.25\end{array}$

5.00

4.75

4.50

.75

.65

$\begin{array}{ll}1.50 & 1.35\end{array}$

1.00

1.25

.90

1.15

.60

1.25

.80

$\begin{array}{lll}1.50 & 1.35 & 1.25\end{array}$

3.00

2.85

2.75

" Verticillatus, Deciduous Holly

Prunus Americana, Wild Plum

1.50

1.00

4.50

“ Lannesiand Japanese Flowering Cherry ....

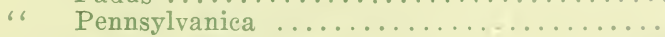

“ Serrulata, Japanese Wild Cherry ..........

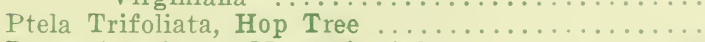

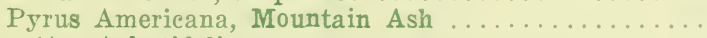

" Arbutifolia

“Aucuparia, European Mountain Ash ........

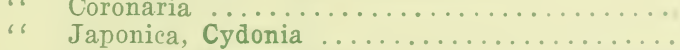

" Rivularis, Oregon Crab

Quercus Alba, White Oak 


\section{DECIDUOUS TREES AND SHRUBS (Continued)}

5 lbs. 25 lbs. 100 lbe.

per oz. per lb, price price price

Quercus Cerris, Turkey Oak

per lb. per lb. per lb.

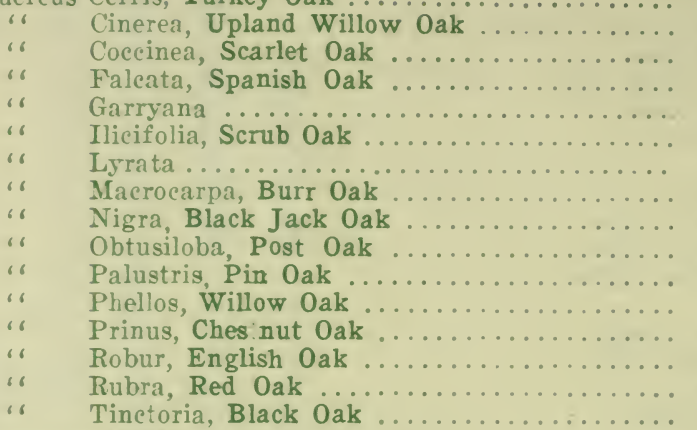

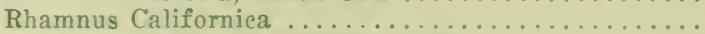

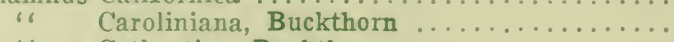

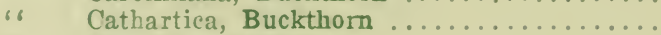

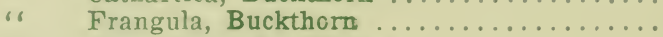

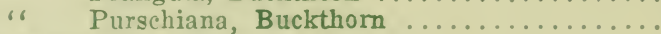

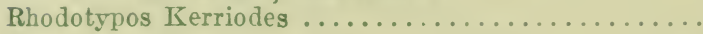

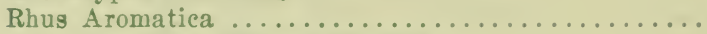

"Copallina, Shining Sumach ............

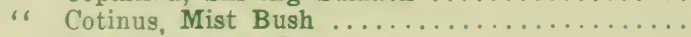

"Glabra, Smooth Sumach .................

“Typhina, Stag's Horn Sumach

.35
.35
.35
.35
1.00
.35
.35
.35
.35
.35
.35
.35
.35
.35
.25
.35

$.30 \quad .25$

$.30 \quad .25$

$.30 \quad .25$

$.30 \quad .25$

$.85 \quad .75$

$.30 \quad .25$

$.30 \quad .25$

$.30 \quad .25$

$.30 \quad .25$

$.30 \quad .25$

$.30 \quad .25$

$.30 \quad .25$

$\begin{array}{ll}.30 & .25\end{array}$

$.30 \quad .25$

$.20 \quad .18$

.20

.20

.20

.20

.15

1.25

.30

.25

.20

.20

.20

.22

.20

.20

.20

.20

.20

.15

$\begin{array}{ll}1.85 & 1.75\end{array}$

$.15 \quad 1.50$

$1.15 \quad 1.00$

.15

.35

1.50

$1.35 \quad 1.25$

1.10

3.50

1.35

1.25

$3.25 \quad 3.15$

3.00

1.25

1.15

1.10

1.00

1.35

1.25

3.50

.65

.60

3.35

3.25

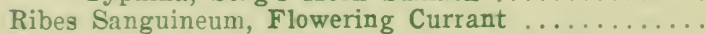

Robinia Pseud-Acacia, Yellow or Black Locust ......

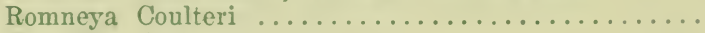

Rosa Blanda

"Canina.

"Carolina

$.50 \quad 3.50$

$.15 \quad .50$

1.0010 .00

$.15 \quad 1.00$

$.15 \quad 1.00$

" Lueida

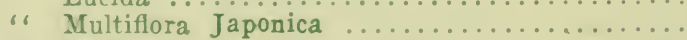

“ Nitida

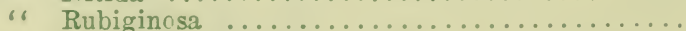

" Rugosa...

.15

“ Setigera, Wild Prairie Rose .............

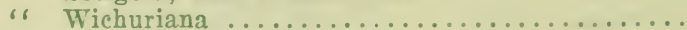

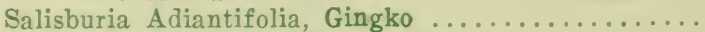

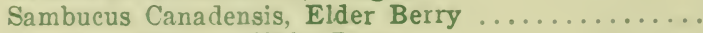

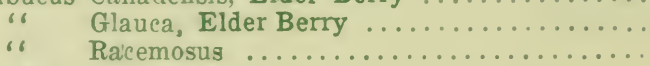

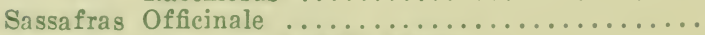

Sheperdia Argentia, Buffalo Cherry ............

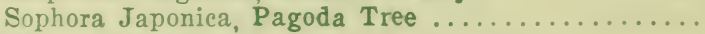

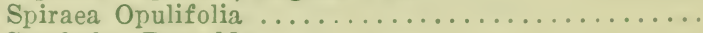

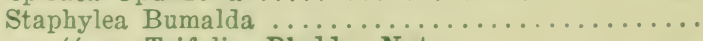

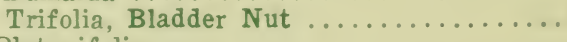

Sterculia Platanifolia

Stuartia Pentagyna

Styrax Japonica

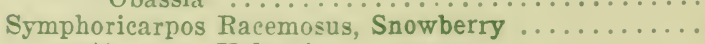

Syringa Vulgaris, Common Lilac (Pods) ................

Taxodium Distichum, Deciduous Cypress ............

Thea Sinensis, Japan Tea Plant 


\section{DECIDUOUS TREES AND SHRUBS (Continued)}

Tilia Americana, American Linden

5 lbs. 25 lbs. 100 lbs.

$\ldots \ldots \ldots \ldots$

s.

6 Europaea, European Linden ..............

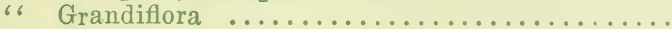

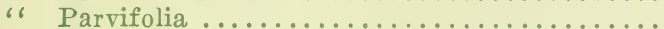

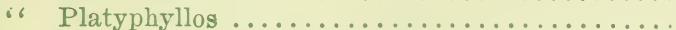

Ulmus Americana, American Elm ..............

"6 Campestre, European Elm ..............

6 Fulva, Slippery Elm ..............

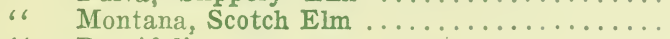

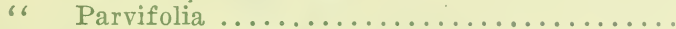

Vaccinium Arboreum, Bush Huckleberry ...........
66 Corymbosum, Bush Huckleberry .........
6. Macrocarpa, Cranberry, Clean Seed ......

"6 Pennsylvanicum, Huckleberry .........

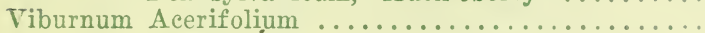

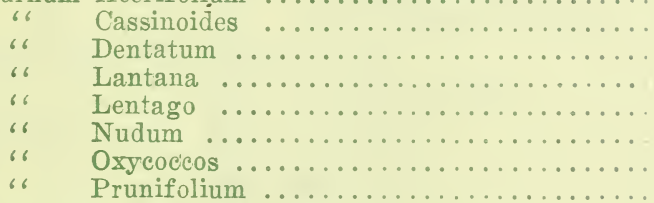

Zanthoxylon Fraxinaefolium, Prickly Ash ........

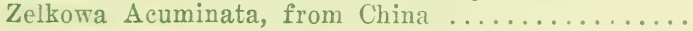

VINES AND CLIMBERS

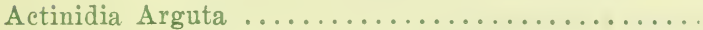

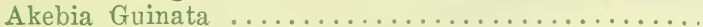

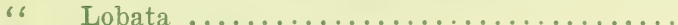

Ampelopsis Heterophylla Variegata, Variegated Grape

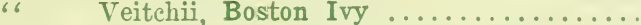
"Veitchii, Boston Ivy, Clean Seed ......

66 Virginica, Virginia Creeper ..........

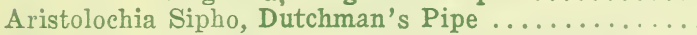

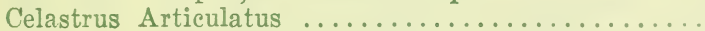
"Scandens, Staff Vine (Dry Berries).......

Clematis Flammula, Sweet Clematis

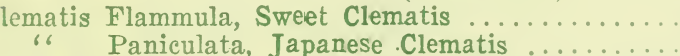

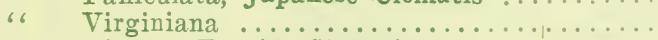

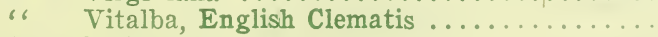

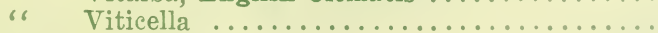

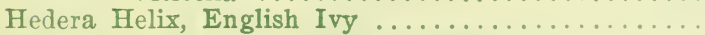

Hydrangea Sceandens, Climbing Hydrangea .........

Memispermum Canadense, Moon Seed ............

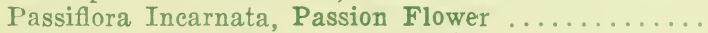

Pueraria Thunbergiana, Kudzu Vine

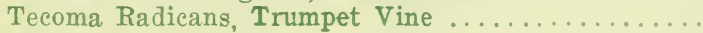

Vitis. See Fruits

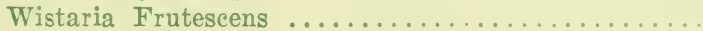

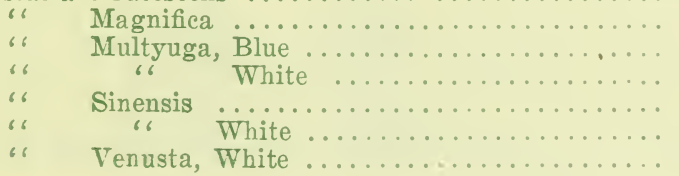

5.00

$.15 \quad 1.50$

$.25 \quad 2.50$

$.15 \quad 1.50$

$1.25 \quad 1.15$

1.00

$.50 \quad 5.00$

1.251 .00

$50 \quad 5.00$

.151 .00

$4.75 \quad 4.50$ 
Color

Boltonia Asteroides, False Chamomile ...........White

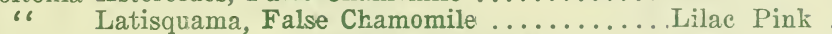

Campanula Calycanthema, Canterbury Bells ......... Blue

$$
\begin{aligned}
& 66 \\
& 66 \\
& 66 \\
& 66 \\
& 66 \\
& 66 \\
& 66 \\
& 66 \\
& 66 \\
& 66 \\
& 66 \\
& 66 \\
& 66 \\
& 66
\end{aligned}
$$

"6

66

" .........Pink

c6

". .......White

6 .........Mixed

Carpatica, Hare Bell ................... Blue

Alba, Hare Bell ........... White

Media, Single Canterbury Bells ..........Blue

"6 "6 "6 ..........Pink

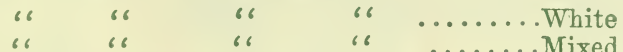

“ Double Canterbury Bells ......... Mixed

Persicifolia Grandiflora, Peach Bells .... Blue

" " Alba, Peach BellsWhite

Pyramidalis, Chimney Bellflower.........Blue

Alba, Chimney Bellflower White

Candytuft, Iberis Sempervirens ............. White

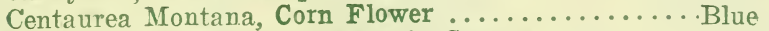

Cerastium Tomentosum, Snow in Summer .........White

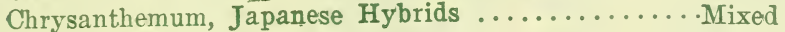

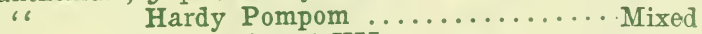

6 I Ting Edward VII ............ White

Coreopsis Lanceolata Grandiflora ....................... Yellow

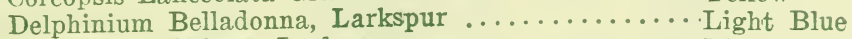

" Chinese Larkspur ................... Chine

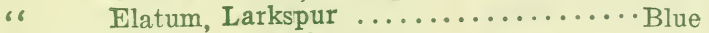

" Formosum, Larkspur ................ Blue

“ Formosum Coelestinum, Larkspur .... Blue

" Kelway's Giant, Larkspur ..................

“ Rev. E. Lascelles, Larkspur ......... Blue

“ Zalil, Larkspur ................ Yellow

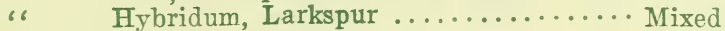

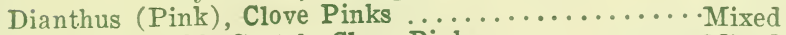

"6 Double Scotch, Clove Pinks ..................

" Erfurt, Clove Pinks Mixed

“ Semperflorens, Everblooming Pinks ....... Mixed

" L Latifolius Abrocoscineus Fl. Pl.

Everblooming Hybrid Sweet William .... Scarlet

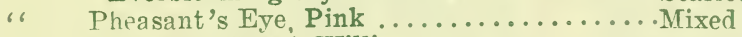

" Barbatus, Sweet William ..........................

" " " Double Sweet William .........Mixed

" " Scarlet Beauty ........................ "

" " Newport Pink .........................

" " $"$ Single ............ Velvety Maroon

$66 \quad 66 \ldots \ldots \ldots \ldots$ White

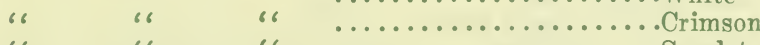

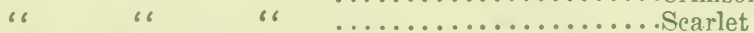

Dictamnus Fraxinella, Gas Plant ..............Red

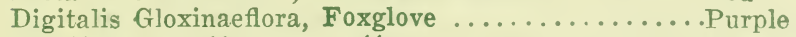

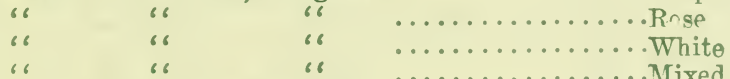

"Grandiflora, Foxglove .................... (ellow

"Monstrosa, Mammoth Foxglove ...........Mixed

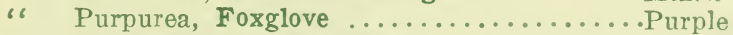

“ Purpurea Alba, Foxglove ............White

Eupatorium Ageratoides, Thorough Wort ........White per Trade per per

pkt. pkt. 1/4 oz. oz.

$\begin{array}{llll}.10 & .25 & .50 & 1.50\end{array}$

$\begin{array}{llll}.10 & .25 & .50 & 1.5 \mathrm{u}\end{array}$

$\begin{array}{llll}.15 & .30 & .75 & 2.00\end{array}$

$\begin{array}{llll}.15 & .30 & .75 & 2.00\end{array}$

$\begin{array}{llll}.15 & .30 & .75 & 2.00\end{array}$

$\begin{array}{llll}.10 & .25 & .50 & 1.50\end{array}$

$\begin{array}{llll}.10 & .20 & .40 & 1.00\end{array}$

$\begin{array}{llll}.10 & .20 & .40 & 1.00\end{array}$

$\begin{array}{llll}.10 & .15 & .25 & .50\end{array}$

$\begin{array}{llll}.10 & .15 & .25 & .50\end{array}$

$\begin{array}{llll}.10 & .15 & .25 & .50\end{array}$

$\begin{array}{llll}.10 & .15 & .25 & .40\end{array}$

$\begin{array}{llll}.10 & .25 & .50 & 1.25\end{array}$

$\begin{array}{llll}.15 & .30 & .100 & 3.00\end{array}$

$\begin{array}{llll}.15 & .30 & 1.00 & 3.00\end{array}$

$\begin{array}{llll}.10 & .25 & .50 & 1.50\end{array}$

$\begin{array}{llll}.10 & .25 & .50 & 1.50\end{array}$

$\begin{array}{llll}.15 & .40 & 1.00 & 2.50\end{array}$

$\begin{array}{llll}.10 & .25 & .50 & 1.00\end{array}$

$\begin{array}{llll}.15 & .30 & .75 & 1.75\end{array}$

$\begin{array}{llll}.25 & .50 & 1.00 & 4.00\end{array}$

$\begin{array}{llll}.25 & .50 & 1.00 & 4.00\end{array}$

$\begin{array}{llll}.10 & .25 & .30 & .75\end{array}$

$\begin{array}{llll}.10 & .15 & .25 & .35\end{array}$

$\begin{array}{llll}.25 & .50 & 1.50 & 3.00\end{array}$

$\begin{array}{llll}.10 & .20 & .30 & .60\end{array}$

$\begin{array}{llll}.10 & .20 & .30 & .60\end{array}$

$\begin{array}{llll}.10 & .25 & .50 & 1.25\end{array}$

$\begin{array}{llll}.10 & .25 & .50 & 1.25\end{array}$

$\begin{array}{llll}.25 & .40 & .75 & 1.75\end{array}$

$\begin{array}{llll}.25 & .40 & .75 & 1.75\end{array}$

$\begin{array}{llll}.30 & .50 & 1.00 & 4.00\end{array}$

$\begin{array}{llll}.25 & .40 & .75 & 3.00\end{array}$

$\begin{array}{llll}.10 & .15 & .25 & .50\end{array}$

$\begin{array}{llll}.15 & .30 & .75 & 2.00\end{array}$

$\begin{array}{llll}.15 & .30 & .75 & 1.75\end{array}$

$\begin{array}{llll}.25 & .40 & .75 & 1.75\end{array}$

$\begin{array}{llll}.25 & .40 & .75 & 1.75\end{array}$

$\begin{array}{llll}.15 & .40 & .75 & 2.00\end{array}$

$\begin{array}{llll}.10 & .15 & .25 & .50\end{array}$

$\begin{array}{llll}.05 & .15 & .20 & .60\end{array}$

$\begin{array}{llll}.10 & .15 & .30 & .75\end{array}$

$\begin{array}{llll}.15 & .25 & .50 & 1.50\end{array}$

$\begin{array}{llll}.15 & .25 & .50 & 1.50\end{array}$

$\begin{array}{llll}.05 & .15 & .20 & .50\end{array}$

$\begin{array}{llll}.05 & .15 & .20 & .50\end{array}$

$\begin{array}{llll}.05 & .15 & .20 & .50\end{array}$

$\begin{array}{llll}.05 & .15 & .20 & .50\end{array}$

$\begin{array}{llll}.10 & .15 & .25 & .60\end{array}$

$\begin{array}{llll}.10 & .20 & .40 & .75\end{array}$

$\begin{array}{llll}.10 & .20 & .40 & .75\end{array}$

$\begin{array}{llll}.10 & .20 & .40 & .75\end{array}$

$\begin{array}{llll}.10 & .15 & .25 & .60\end{array}$

$\begin{array}{llll}.10 & .15 & .25 & .60\end{array}$

$\begin{array}{llll}.10 & .25 & .50 & 1.00\end{array}$

$\begin{array}{llll}.10 & .25 & .50 & 1.00\end{array}$

$\begin{array}{llll}.10 & .25 & .50 & 1.00\end{array}$

$\begin{array}{llll}.10 & .25 & .50 & 2.00\end{array}$ 
Elipatorium Coelstinum, Thorough wort ......... Lavender

Fraseri, Thorough Wort ...........White

Gaillardia Grandiflora Compacta, Blanket Flower ...Mixed

"6 " 6 $\quad$ Sanguinea, Blanket Flower ...Red

66

Semi-Double, Blanket Flower. .Mixed

Superba, Blanket Flower .....Mixed

Kermesian Splendius, Blanket Flower .... Mixed

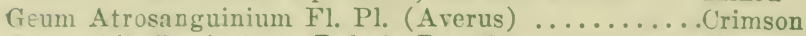

Gypsophila Paniculata, Baby's Breath ...........White

Fl. Pl. Baby's Breath, DoublcWhite

Helenium Autumnale Superbum, Sneeze Wort ........ Yellow

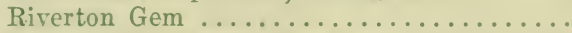

Heliopsis Pitcheriana, Orange Sunflower ........... Yellow Scabra Zinniaeflora, Orange Sunflower .... Tellow

per Trade per per
pkt. pkt. $3 / 4$ oz. oz.

$\begin{array}{llll}15 & 30 & 50 & 200\end{array}$

$\begin{array}{llll}.10 & .15 & .40 & .75\end{array}$

$\begin{array}{llll}.10 & .15 & .25 & .60\end{array}$

$\begin{array}{llll}.15 & .20 & .40 & .75\end{array}$

$\begin{array}{llll}.15 & .20 & .40 & .75\end{array}$

$\begin{array}{llll}.10 & .15 & .25 & .60\end{array}$

$\begin{array}{llll}.15 & .30 & .50 & 1.50\end{array}$

$\begin{array}{llll}.10 & .20 & .40 & .75\end{array}$

$\begin{array}{llll}.10 & .15 & .25 & .50\end{array}$

$\begin{array}{llll}.35 & .50 & 2.00 & 7.50\end{array}$

$\begin{array}{llll}.10 & .25 & .50 & 1.50\end{array}$

$\begin{array}{llll}.10 & .25 & .50 & 2.00\end{array}$

$\begin{array}{llll}.10 & .15 & .40 & .60\end{array}$

$\begin{array}{llll}.15 & .25 & .50 & 2.00\end{array}$

$\begin{array}{llrrr}\text { Heuchera Sanguinea, Crimson } \ldots \ldots \ldots \ldots \ldots \ldots \ldots \ldots \ldots \text { Crimson } & .25 & .40 & 1.00 & 2.25 \\ \text { Hibiscus Crimson Eye, Marshmallow................. \& C Crimson } & .10 & .15 & .40 & .75\end{array}$

Mescheutos, Marshmallow

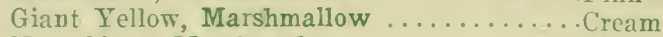

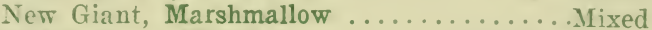

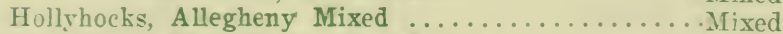

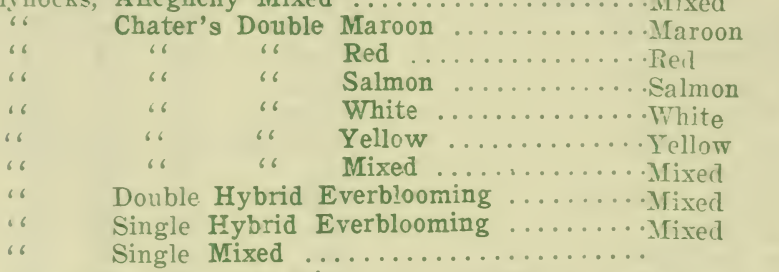

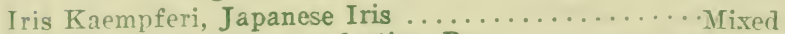

Lathyrus Latifolius, Everlasting Pea ............. r rimson

\begin{tabular}{|c|c|c|c|c|}
\hline & 66 & 66 & 66 & Pink Beauty.Pink \\
\hline 66 & "6 & 66 & 66 & White \\
\hline 66 & "6 & “ & 16 & $\cdots \mathrm{M}$ \\
\hline
\end{tabular}

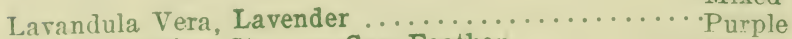

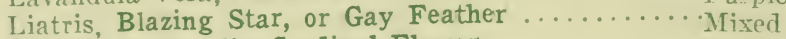

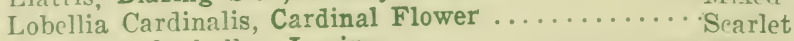

Lupinus Polyphyllus, Lupine ......................

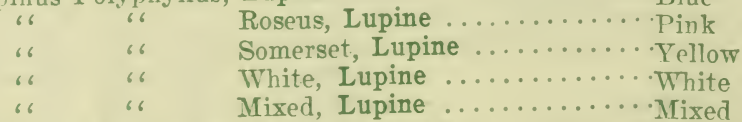

Lychnis Chalcedonica, Jerusalem Cross ......................

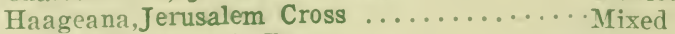

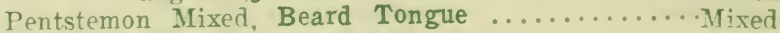

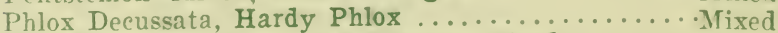

Physostegia Virginica, False Dragon Head .........Pink

Alba, False Dragon Head ... White

Platycodon Grandiflora, Japanese Bellflower ....... Blue

Alba, Japanese Bellflower . White

Mariesi, Japanese Bellflower .......... Tinlet-Blue

Japonicus Fl. Pl., Double Jap. Bellflower. Blue

Polyanthus Gold-laced, Primula Elatior .........................

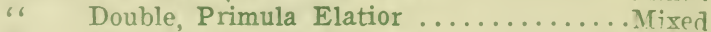

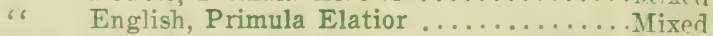

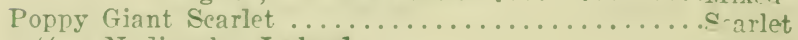

Nudicaule, Iceland ..............White

Yellow

Doub!e

Mixed
.10

.10

.10

.10

.10

.10

.10

.10

.10

.10

.15

.15

.10

.10

.10

.10

.10

.10

.10

.10

.15

.10

.15

.15

.10

.10

.10

.15

.10

.10

.10

.10

.10

.10

.10

.15

.20

.25

.10

.10

.15

.15

.15
.15

.15

.20

.20

.20

.20

.20

.20

.20

.20

.25

.20

.20

.15

.15

.20

.15

.15

.15

.25

.50

.15

.20

.30

.15

.15

.15

.40

.25

.30

.20

.25

.25

.25

.25

.40

.40

.50

.25

.15

.25

.25

.25
$.25 \quad .50$

$\begin{array}{ll}.50 & 1.60\end{array}$

$.30 \quad .60$

$\begin{array}{ll}.30 & .75\end{array}$

$\begin{array}{ll}.30 & 1.00\end{array}$

$\begin{array}{ll}.30 & 1.00\end{array}$

$\begin{array}{ll}.30 & 1.00\end{array}$

$\begin{array}{ll}.30 & 1.00\end{array}$

$\begin{array}{ll}.30 & 1.00\end{array}$

$.30 \quad .60$

$\begin{array}{ll}.50 & 1.00\end{array}$

$.30 \quad .60$

$.30 \quad .60$

$.25 \quad .50$

$.30 \quad .75$

$.40 \quad 1.25$

$.30 \quad .75$

.25 .60

$.30 \quad .75$

$\begin{array}{ll}.50 & 2.00\end{array}$

$1.25 \quad 4.00$

$.25 \quad .40$

$\begin{array}{ll}.35 & .75\end{array}$

$\begin{array}{ll}.75 & 2.00\end{array}$

$.25 \quad .40$

$.25 \quad .30$

$.25 \quad .40$

$.75 \quad 2.00$

$.50 \quad 1.75$

$.60 \quad 1.75$

$\begin{array}{ll}.30 & .75\end{array}$

$\begin{array}{ll}.50 & 1.25\end{array}$

$.40 \quad 125$

$40 \quad 1.25$

$\begin{array}{ll}.50 \quad 2.00 \\ .85 & 2.50\end{array}$

$.85 \quad 2.50$

$.85 \quad 2.50$

$1.00 \quad 4.00$

$.60 \quad 2.00$

$.25 \quad .50$

$\begin{array}{ll}.50 & 1.25\end{array}$

$\begin{array}{ll}.50 & 1.25\end{array}$

$\begin{array}{ll}.50 & 1.25\end{array}$ 


\section{"Mutual Benefit"}

The price of The Farm Journal is $\$ 1.00$ for 4 years; but since we believe that to read this wide-awake farm and household paper is the greatest benefit to our friends and patrons, and so indirectly to us, we have persuaded the publishers to

make us this

\section{SPECIAL TRIAL RATE OF}
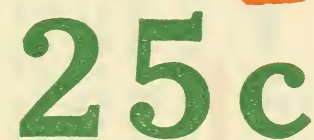

for a year's trial of the paper.

The Farm Journal is published monthly; 64 to 168 pages of new, reliable, practical, up-to-date articles and fine pictures. 45 years old, has over $5,500,000$ readers from Maine to California.

"UNLIKE ANY OTHER PAPER"

Full of Gumption-Full of Sunshine

Care of stock, horses, cows, sheep, swine; big poultry department; soil building and fertilizers; all about tractors, engines, autos; orchard, garden, vegetables; insect pests and how to control them: big Bird Club; pages for young people; dressmaking, fashions, patterns, embroidery; new economy recipes; farm law; beautiful poetry, Walt Mason's "poems in prose"; absorbing stories of love, adventure, and mystery.

Mail this Mutual Benefit Coupon today with twentyfive cents, silver or stamps, direct to The Farm Journal. DO NOT SEND IT TO US.

CONTERS B. FLEU, JR. Seedsman

6628-30-32 Ross St., Germantown, Phila. Pa. 


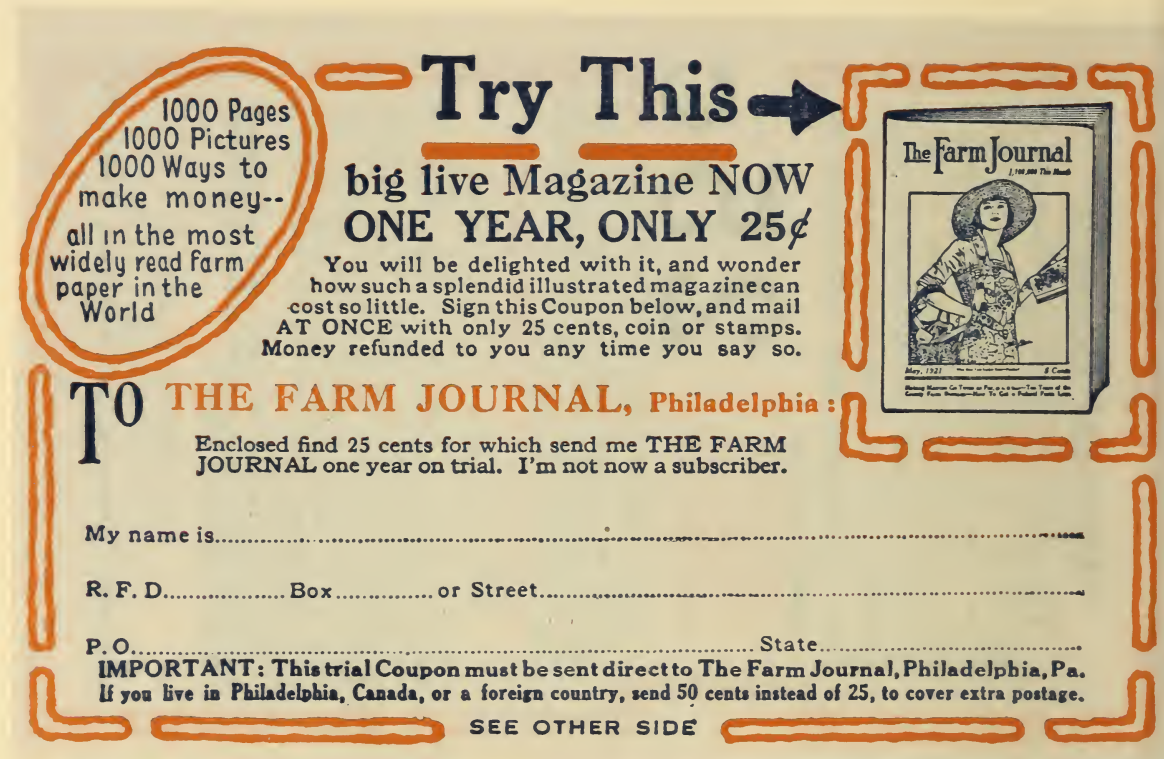




\section{Color}

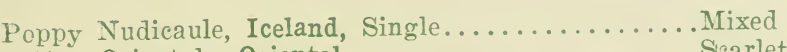
" Orientale, Oriental

"6 6 "6 Mrs. Perry, Oriental ...

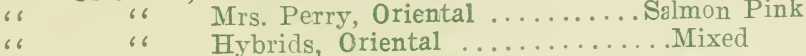

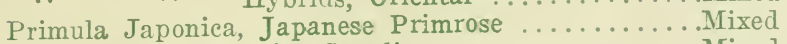

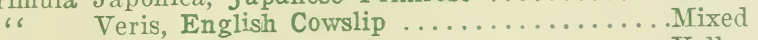

“Vulgaris, English Primrose ............ Yellow

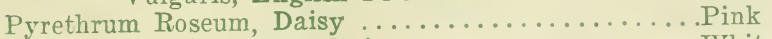

، Uliginosum, Daisy .............White

، Hybridum, Single Daisy .............................

" Hybridium, Fl. Pl. Double Daisy ..........Mixed

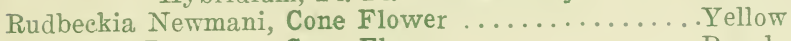

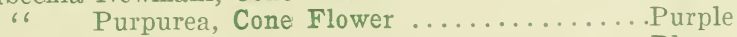

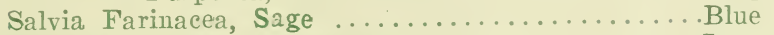

Scabiosa Caucasia, Blue Bonnet ...................................
66
Alba, White Bonnett
White
Japonica,
Japanese Bonnett
Blue

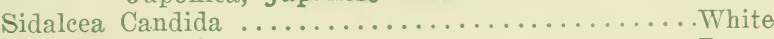

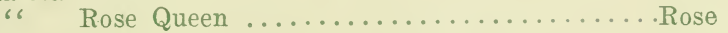

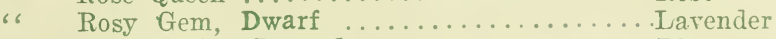

Statice Latifolia, Sea Lavender .............. Blue

Stokesia Cyanea, Cornflower Aster ............... Blue

"6 Alba, Cornflower Aster .........White

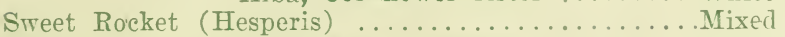

Sunflower (Helianthus), Golden Bouquet .......... Yellow

"6 "Choice Mixed ...........Mixed

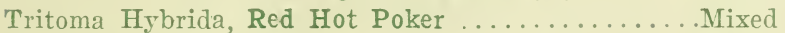

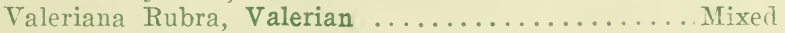

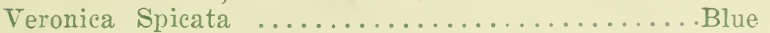

$\begin{array}{cccc}\text { per } & \text { Trade } \\ \text { pkt } & \begin{array}{c}\text { per } \\ \text { pkt } \\ 1 / 4 \\ \text { oz. }\end{array} & \begin{array}{c}\text { per } \\ \text { oz. }\end{array} \\ .10 & .15 & .25 & .75 \\ .10 & .15 & .30 & .75 \\ .25 & .40 & .90 & 2.50 \\ .15 & .25 & .50 & 1.25 \\ .15 & .25 & .50 & 1.75 \\ .15 & .25 & .50 & 1.50 \\ .15 & .25 & .50 & 2.09 \\ .25 & .40 & .75 & 2.00 \\ .25 & .40 & .75 & 3.00 \\ .20 & .30 & .50 & 1.50 \\ .25 & .50 & 1.00 & 400 \\ .15 & .30 & .50 & 1.50 \\ .15 & .30 & .50 & 1.25 \\ .10 & .25 & .50 & 1.25 \\ .15 & .40 & .75 & 2.25 \\ .15 & .40 & .75 & 2.25 \\ .10 & .15 & .30 & .75 \\ .10 & .15 & .50 & 2.00 \\ .15 & .40 & .75 & 3.00 \\ .10 & .15 & .50 & 2.00 \\ .10 & .25 & .50 & 1.25 \\ .10 & .25 & .50 & 1.25 \\ .10 & .25 & .50 & 1.25 \\ .05 & .15 & .20 & .30 \\ .10 & .25 & .50 & 2.00 \\ .10 & .25 & .50 & 2.00 \\ .15 & .25 & .50 & 2.00 \\ .10 & .15 & .35 & .60 \\ .15 & .25 & .40 & .125 \\ & & & \end{array}$

\section{HORTICULTURAL BOOIKS}

THE NURSERY MANUAL: By Prof. L. H. Bailey. Founded on the Nursery Book, but entirely rewritten and re-illustrated. This book is a complete guide to the

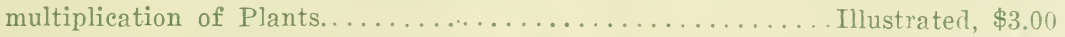

BAILEY'S STANDARD CYCLOPEDIA OF HORTICULTURE: By Prof. L. H. Bailey. By four hundred leading botanists and horticulturists of North America, each selected as the expert of the highest reputation in his specialty, under the general editorship of L. H. Bailey. Six large quarto volumes, over 3,600 pages, 24 full page color plates, 96 special full-page half tones, more than 4,000 text engravings, approximately 3,300 general, 20,000 species and 40,000 plant names. Each State in the Union has separate treatment and there are special articles on Canada; Porto Rico; Hawaii and the Philippines. It is the most modern work of its kind and should be in the library of every progressive horticulturalist. Now complete in 6 Vols. Set $\$ 48.00$.

THE SPRAYING OF PLANTS: By E. G. Lodeman. Full directions regarding materials and formulas for spraying, with deseription of apparatus used. Each flower and fruit with respective forms of blight and insect is discussed individ

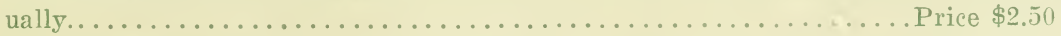

THE PRUNING MANUAL: By L. H. Bailey. Revised and reset, this book incorporates the results of fifteen years of experience in pruning. Pruning in all its varied phases is thoroughly handled. Revised and Rewritten. Illustrated. .\$3.00 


\section{FERTILIZERS}

This list of Fertilizers should hava your careful consideration in preparing your seed beds.

Prices are F. O. B. Germantown.

BONE MEAL: The Fertilizer for all purposes as it contains two very rich and important plant foods: namely, nitrogen and Phosphoric acid. 5 lbs. $\$ .30 ; 10 \mathrm{lbs}$. $\$ .50 ; 25 \mathrm{lbs} . \$ 1.25 ; 50$ lbs. $\$ 2.25 ; 100$ lbs. $\$ 4.00 ; 200$ lbs. $\$ 7.25 ; 1 / 2$ ton $(1000$ lbs.) $\$ 33.50 ; 1$ ton $(2000)$ lbs.) $\$ 65.00$.

HUMUS: (Alphano Prepared). Humus should always be liberally mixed with the soil when preparing your hotbeds or seed beds. It lightens heavy soil and makes it more porous. It is especially good for starting seeds and bulbs as it cannot burn or injure the plants. 5 lbs. $\$ .25 ; 10$ lbs. $\$ .40 ; 25$ lbs. $\$ .75 ; 100$ lbs. $\$ 2.50 ; 500$ lbs. $\$ 11.25 ; 1000$ lbs. $\$ 17.00 ; 1$ ton (2000 lbs.) $\$ 30.00$.

SHEEP MANURE: (Wizard Brand). A pulverized and natural manure. Nothing surpasses it for mixing with the soil as it has all of the necessary plant food requirements and gives immediate results. $5 \mathrm{lbs} . \$ .40 ; 10 \mathrm{lbs} . \$ .70 ; 25 \mathrm{lbs} . \$ 1.00$; 50 lbs. $\$ 1.75 ; 100$ lbs. $\$ 3.40 ; 500$ lbs. $\$ 15.00 ; 1000$ lbs. $\$ 28.00 ; 1$ ton (2000 lbs.) $\$ 55.00$.

\section{INSECT DESTROYERS}

\section{Directions on Each Package}

ARSENATE OF LEAD: (Powdered). The best spray to destroy leaf chewing insects; also prevents the codling moth destroying Fruit. Per 1b. $\$ .55 ; 5$ lbs. $\$ 2.50 ; 10$ lbs. $\$ 4.50 ; 25$ lbs. $\$ 10.50 ; 50$ lbs. $\$ 19.00 ; 100$ lbs. $\$ 36.00$.

BORDEAUX-ARSENATE LEAD: (Paste). Best for mildew, Blight and Fungous diseases. Per lb. $\$ .40 ; 5$ lbs. $\$ 1.50 ; 10$ lbs. $\$ 2.50 ; 25$ lbs. $\$ 5.25 ; 50$ lbs. $\$ 9.00$; 100 lbs. $\$ 17.00$.

BORDEAUX MIXTURE: (Paste) Same price as above.

FISH OIL SOAP: (Good's Caustic). Spray Evergreens for the red spider and your trees in summer for scale. An excellent wash and spray for Greenhouse Plants. Per lb. $\$ .25 ; 5$ lbs. $\$ .95 ; 25$ lbs. $\$ 4.25 ; 100$ lbs. $\$ 15.00$.

KEROSENE EMULSION: (Liquid). Will destroy mild form of seale and soft lice. Per qt. $\$ .80 ; 1$ gal. $\$ 2.00 ; 5$ gal. $\$ 7.50$.

LIME SULPHUR: An excellent winter or early spray for scale or Fungous diseases that attack Fruit Trees, Deciduous Trees and Shrubs. Per 1/2 Gal. \$.70; 1 gal. $\$ 1.00$; 5 gals. $\$ 3.75$; $1 / 2 \mathrm{Bbl}$. (30 gals.) $\$ 10.75$; per Bbl. (50 Gals.) $\$ 15.00$.

SCALECIDE: Spray your Fruit, Shrubs and Trees with Scalecide when they are dormant. It will destroy San Jose Scale and means better Fruit and stronger plants. Per qt. $\$ .60$; per gal. $\$ 1.45 ; 5$ gal. $\$ 6.45 ; 10$ gal. $\$ 11.00 ; 30$ gal. $\$ 27.00$; 50 gal. $\$ 39.50$. 